\title{
Dialisi peritoneale: dialisi marginale? 2013 Tentativo di sintesi delle opinioni a confronto
}

\author{
Gianna d'Adamo
}

Nefrologa, Roma

\begin{abstract}
Peritoneal dialysis: MARginal dialysis? 2013 An ATTEMPT to SYNTHETIZE THE OPINIONS OF THE EXPERTS
Abstract. Summary of the opinions expressed by Italian experts in the last special issue of this journal dedicated to the question of whether peritoneal dialysis (PD) is still marginal.

The PD rate in Italy is stably around $10 \%$, due to the frequently inadequate training of Nephrologists, poor organization of the Nephrology units, low reimbursements, regulatory gaps, and the existence of many private centers not authorized to use PD. Other factors that hinder PD penetration are the increasing number of frail elderly patients starting dialysis, and the risk of sclerosing peritonitis, at least in the long run. The need for good quality pre-dialysis education and further progress of research are here strongly emphasized.
\end{abstract}

Key words: Peritoneal dialysis, Epidemiology of peritoneal dialysis

Conflict of Interests: None.

Financial support: None.

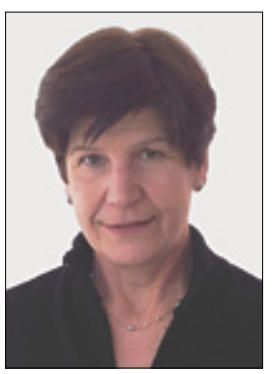

Gianna d'Adamo
L'ultimo numero speciale di questo Giornale è dedicato a rispondere alla domanda se la dialisi peritoneale (DP) sia ancora oggi una dialisi marginale. Molti colleghi "peritonealisti" hanno risposto alla sollecitazione di un"“analisi libera, spontanea, anche provocatoria ma, soprattutto, non preordinata" dell'Editor, che già aveva curato l'analoga iniziativa del 2001. Catizone crede amaramente, e io con lui, che questo nuovo numero speciale non sarà letto dai colleghi "emodializzatori” e/o dagli amministratori e/o dai politici e che, quindi, si continuerà a parlare dell'argomento solo tra "peritonealisti". Da qui, il tentativo di una breve sintesi delle molte opinioni espresse che, cercando di non travisarne la ricchezza e l'articolazione, invogli alla lettura soprattutto i colleghi nefrologi che non praticano la dialisi peritoneale. In Italia, la percentuale di pazienti prevalenti in dialisi peritoneale si situa intorno al $10 \%$, stabile da molti anni e inferiore a quanto sarebbe "giusto", secondo l'opinione di molti; corrispondente alla nicchia di pazienti che effettivamente ne traggono vantaggio, secondo altri. La DP è, dunque, stabilmente "marginale" e stabile è anche buona parte delle motivazioni ritenute alla base del suo scarso utilizzo, che vanno dall'inadeguata formazione universitaria dei nefrologi alla carente organizzazione interna delle unità operative e dalla miopia degli amministratori alle tariffe penalizzanti per i rimborsi e all'alto numero di Centri Privati non autorizzati a effettuare la DP. A ciò si aggiungono il progressivo invecchiamento della popolazione uremica, con la necessità di immettere in dialisi persone anziane, spesso affette anche da altre patologie e spesso non autosufficienti, sole o con uno scarso supporto familiare, e, soprattutto, la maggiore consapevolezza di parte dei nefrologi della non-fisiologicità del trattamento e del rischio di peritonite sclerosante, almeno nel lungo periodo. Il peso relativo di queste motivazioni varia nei diversi Centri e da Regione a Regione. Basti pensare che ci sono ancora Centri Pubblici che non hanno un programma di dialisi peritoneale e che la prevalenza della dialisi peritoneale in Campania si situa intorno al $2 \%$. Qualcosa, dal punto di vista normativo, è stato fatto: in Sicilia e in Piemonte, dove i nefrologi sono riusciti a farsi ascoltare, la Regione ha preso in seria considerazione il possibile risparmio derivante dall'immissione in dialisi peritoneale di un maggior numero di pazienti, tanto da deliberare l'erogazione di un contributo economico a chi sceglie di fare la dialisi a domicilio. Il provvedimento non ha, però, determinato i risultati sperati e l'incremento maggiore si è avuto in Piemonte solo quando sono stati posti anche obiettivi di in- 
cidenza e prevalenza per i direttori generali. Evidentemente, l'atto amministrativo, da solo, non ha potuto districare il complicato intreccio degli altri fattori che condizionano il ricorso alla DP. Un maggiore stimolo potrebbe, però, arrivare da decisioni regionali di più ampio respiro, come la recente adozione, sempre in Piemonte, del PDTA sull'avvio del trattamento sostitutivo e sul consolidamento delle attività relative all'ambulatorio per la malattia renale avanzata. L'importanza dell'ambulatorio dedicato alla malattia renale avanzata, o dell'uremia o di predialisi, è stata richiamata nella maggior parte delle analisi dedicate alla marginalità della DP. E, in primo luogo, una risposta strutturata al dovere etico informare malati e familiari su caratteristiche, vantaggi e limiti delle terapie sostitutive, ma è anche una modifica dell'organizzazione del lavoro dell' equipe nefrologica che potrebbe incidere sull'atteggiamento culturale dei professionisti. Infatti, lo scetticismo di molti nefrologi verso la DP, che spesso consegue a carenze formative durante la scuola di specializzazione, si consolida all'interno di unità operative dove l'organizzazione penalizza la metodica e dove c'è un solo medico che "crede" nella DP. Non a caso molti "peritonealisti" sottolineano la necessità di una grande dedizione personale e l'isolamento che si prova tra colleghi con un diverso livello di competenza nella gestione delle complicanze o anche solo di necessità cliniche non programmate. Lo stesso accade all'interno della componente infermieristica dell'equipe. L'ambulatorio dedicato è anche il luogo per individuare, con la collaborazione di diverse figure professionali, la maniera migliore per affrontare nel singolo caso le difficoltà derivanti dai cambiamenti demografici e sociali. Per sostenere l'eventuale scelta della DP, c'è però, generalmente, poco o nulla da proporre in termini di servizi a domicilio e dialisi assistita o in RSA. La novità più importante rispetto alle opinioni espresse nel 2001 è il maggiore rilievo attribuito alla scarsa biocompatibilità del trattamento e al rischio di peritonite sclerosante (EPS). Con forza e autorevolezza, Di Paolo e Buoncristiani sottolineano la possibilità di danni gravissimi e spesso irreparabili del peritoneo, a seguito del contatto con sostanze estranee, e invitano a riconsiderare l'impiego della DP come trattamento di prima scelta. Il gruppo di Brescia, invece, considera l'EPS una complicanza che influenza la diffusione della metodica prevalentemente in termini di pregiudizio. Per i pediatri del Policlinico di Milano, l'aumento del rischio di EPS con l'aumento dell'età dialitica a seguito del prolungamento del tempo di attesa per il trapianto è uno dei motivi per cui l'assunto "bambino in dialisi = bambino in peritoneale" non deve più essere considerato come assoluto. In conclusione, l'insieme dei contributi a questa nuova analisi delle problematiche connesse con l'uso della DP riveste un interesse anche superiore rispetto alle attese. Assume grande evidenza la responsabilità ineludibile del nefrologo circa i contenuti dell'informazione al paziente su vantaggi e limiti delle diverse terapie sostitutive, ma emerge prepotentemente anche la necessità di ulteriori passi in avanti della ricerca per migliorare la metodica o, almeno, il suo utilizzo.

\section{Riassunto}

Breve sintesi delle molte opinioni espresse dai "peritonealisti" italiani nell'ultimo numero speciale di questo Giornale, dedicato a rispondere alla domanda se la dialisi peritoneale (DP) sia ancora marginale.

Lo scarso utilizzo della DP in Italia, stabile da molti anni intorno al $10 \%$, è attribuito a motivi che vanno dall'inadeguata formazione universitaria dei nefrologi alla carente organizzazione interna delle unità operative e dai rimborsi non adeguati ai vuoti normativi ai molti Centri Privati non autorizzati a effettuare la DP.

Altri fattori che ostacolano la diffusione della metodica sono il progressivo invecchiamento della popolazione che inizia la dialisi e il rischio di peritonite sclerosante, almeno nel lungo periodo.

Si sottolineano l'importanza dell'informazione al paziente su vantaggi e limiti delle diverse terapie sostitutive e la necessità di ulteriori passi avanti della ricerca per migliorare la metodica o, almeno, il suo utilizzo.

Parole chiave: Dialisi peritoneale, Epidemiologia della dialisi peritoneale

Dichiarazione di conflitto di interessi: L'Autore dichiara di non avere conflitto di interesse.

Contributi economici agli Autori: L'Autore dichiara di non aver ricevuto sponsorizzazioni economiche per la preparazione dell'articolo.

\section{Indirizzo dell'Autore:}

Dr.ssa Gianna d'Adamo

Nefrologa

Roma

giannadadamo@gmail.com

\section{Per approfondire}

-Dialisi peritoneale: dialisi marginale? 2013 - opinioni a confronto, http://www.gtnd-online.com/pub/gtnd-commenti-dp. -Dialisi peritoneale - commenti aperti, http://www.gtnd-online.com/pub/gtnd-dp---commenti-aperti. 